\title{
Ergebnisse nach Mikro-TESE und ICSI
}

Das Klinefelter-Syndrom (Karyotyp 47, XXY) ist die häufigste Ursache für eine nicht obstruktive Azoospermie. Verschiedene Autoren haben bei den Partnerinnen dieser Männer bei Einsatz moderner reproduktionsmedizinischer Methoden erfolgreiche Schwangerschaften beschrieben. Eine Gruppe aus Teheran berichtet über ihre Erfahrungen mit der mikrochirurgischen testikulären Spermagewinnung (Mikro-TESE) bei diesen Patienten. Urology 2014; 83: 107-110

\section{mit Kommentar}

Die Mikro-TESE mit anschließender intrazytoplasmatischer Spermieninjektion (ICSI) ist bei Männern mit KlinefelterSyndrom (KS) mindestens ähnlich erfolgreich wie bei Männern mit normalem Karyotyp.

Zu diesem Ergebnis kommen Marjan Sabbaghian und Kollegen, die retrospektiv die Daten von insgesamt 134 Patienten mit reinem, gesichertem Klinefelter-Syndrom (keine Mosaike) ausgewertet haben. Als
Kontrollgruppe dienten 537 Männer mit nicht obstruktiver Azoospermie anderer Genese.

Bei allen Patienten erfolgte die Spermiengewinnung zwischen 2009 und 2011 in einem Teheraner Zentrum. Die ovarielle Stimulation und Eizellgewinnung bei den Frauen fand nach dem üblichen Protokoll statt, die ICSI nach der von Van den Bergh beschriebenen Technik.

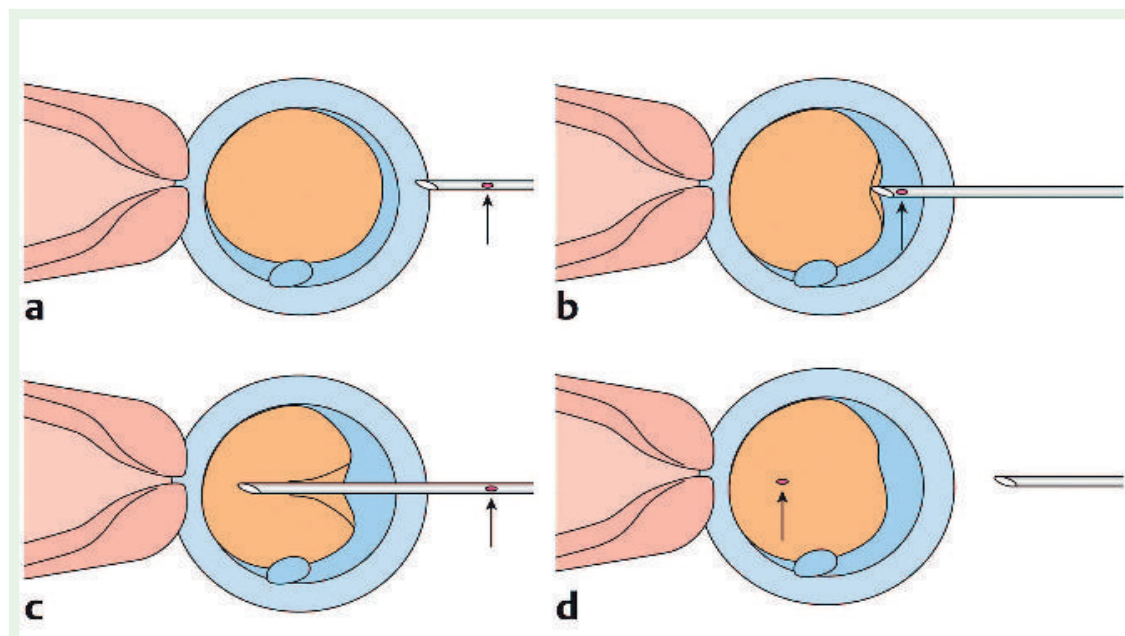

Intrazytoplasmatische Spermieninjektion (ICSI).

a Die Eizelle wird mit dem Polkörper in 6-Uhr-Position durch die Haltepipette links fixiert.

b Die Injektionspipette mit dem Spermium im Spitzenbereich (Pfeil) penetriert die Zona pellucida. c Das Oolemm wölbt sich zeltartig über die Spitze der Injektionspipette. Durch Aspiration von Zytoplasma wird das Spermium von der Spitze in das Innere der Injektionspipette verlagert (Pfeil). d Nach Injektion des Inhalts kommt das Spermium im Zytoplasma der Eizelle zu liegen (Pfeil). (Bild: Kleinstein J, Schuppe HC. Assistierte Fertilisierung. In: Krause W, Weidner W, Sperling H, Diemer T, Hrsg. Andrologie. 4.Aufl. Stuttgart: Thieme; 2011)
Beurteilt wurden

- die Ausbeute bei der Mikro-TESE,

- Faktoren, die mit einem Erfolg dabei verbunden waren,

- die Anzahl der übertragenen Embryos und der Lebendgeburten.

Die Auswertung ergab eine erfolgreiche Spermiengewinnung bei $28,4 \%(n=38)$ der Männer in der Klinefelter-Gruppe und bei $22,6 \%$ in der Kontrollgruppe $(n=119)$, was keinem signifikanten Unterschied entsprach. Allerdings waren bei den Klinefelter-Männern die Patienten, bei denen die Mikro-TESE erfolgreich verlief, signifikant jünger als die Patienten, bei denen das nicht der Fall war (30,8 vs. 33,7 Jahre). Ebenso waren die Partnerinnen dieser Männer deutlich jünger (25,9 vs. 29,1 Jahre). Zudem war die Testosteronkonzentration bei den Klinefelter-Männern mit erfolgreicher Mikro-TESE signifikant höher als bei den Männern ohne ausreichende Spermatozoengewinnung $(3,4$ vs. $2,33 \mathrm{ng} / \mathrm{ml})$. In der Vergleichsgruppe dagegen zeigten sich keine solchen Unterschiede.

Eine logistische Regressionsanalyse, in die FSH-, LH- und Testosteronkonzentrationen sowie Alter und Patientengruppe (Klinefelter vs. Kontrollen) eingingen, ergab keinen der untersuchten Faktoren als signifikanten Prädiktor für eine erfolgreiche TESE.

Die Fertilisationsrate lag in der Klinefelter-Gruppe höher als in der Kontrollgruppe ( 28 vs. $21 \%$ ), und insgesamt 5 Kinder aus 4 Schwangerschaften kamen in dieser Gruppe lebend zur Welt (13 vs. 3\% pro Embryotransfer in der Vergleichsgruppe).

\section{Fazit}

Auch bei Männern mit Klinefelter-Syndrom ist eine erfolgreiche Kinderwunsch-Behandlung mittels Mikro-TESE und anschließender ICSI möglich, so die Autoren, und hat nicht weniger Aussichten auf Erfolg als bei Männern mit einer nicht obstruktiven Azoospermie anderer Genese. Dabei wurden die Embryos vor dem Transfer nicht auf chromosomale Abweichungen getestet. Dies plant die Gruppe der Verfasser in weiteren Untersuchungen.

\section{Dr. Elke Ruchalla, Trossingen}




\section{Kommentar}

\section{Ermutigendes Ergebnis für Patienten mit KS}

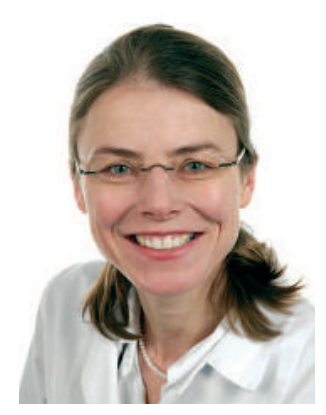

Dr. Julia Rohayem ist Fachärztin für Kinderund Jugendmedizin, Päd. Endokrinolgin / Diabetologin und Andrologin am Centrum für Reproduktionsmedizin und Andrologie am Universitätsklinikum Münster

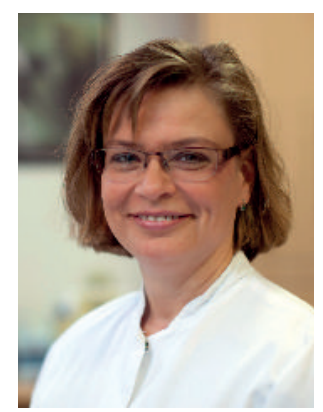

Prof. Dr. Sabine Kliesch ist Chefärztin am Centrum für Reproduktionsmedizin und Andrologie am Universitätsklinikum Münster

Die Studie aus Teheran vergleicht ein großes Patientenkollektiv von 434 Patienten mit einem gesicherten Klinefelter-Syndrom (KS) ohne Mosaik mit 537 Männern mit nicht obstruktiver Azoospermie (NOA) bezüglich des Erfolgs einer mikrochirurgischen testikulären Spermienextraktion (mTESE) bzw. einer intrazytoplasmatischen Spermieninjektion (ICSI).

Die Altersgruppen der verglichenen Patienten sind ähnlich: Das mittlere Alter in der KS-Gruppe lag bei 30 Jahren versus 33,64 Jahren in der NOA-Gruppe. Dahingegen ist das Alter der Partnerinnen in den Gruppen deutlich unterschiedlich: In der KS-Gruppe waren die Partnerinnen mit 25,94 Jahren deutlich jünger als in der NOA-Gruppe (29,0 Jahre). Dies ist für den Erfolg des Embryonentransfers relevant und hat möglicherweise Einfluss auf das Ergebnis der berichteten Lebendgeburten, die bei $13 \%$ (KS) versus 3\% (NOA) lagen. Die Studie liefert keine Daten, ob es sich um gesunde Neugeborene handelt und ob eine postnatale Karyotypisierung erfolgte.

Trotz der genannten Einschränkungen ist dieses Ergebnis für Patienten mit Klinefelter-Syndrom sehr ermutigend, berück- sichtigt man, dass bei der Nicht-MosaikForm dieses Syndroms eine Chromosomenanomalie mit einem überzähligen XChromosom in allen somatischen Zellen und zum großen Teil auch den Keimzellen vorliegt. Nur wenige Keimzellen sind euploid; von diesen Zellen kann eine intakte Spermatogenese ausgehen. Im Vergleich zu den Ergebnissen anderer Forschergruppen liegt die Spermien-Asservierungsrate in der hier vorgestellten Arbeit bei ca. 30\%. Beim KS variieren die in der Literatur berichteten Erfolgsraten zwischen 21 und 72\% [1, 2]. Von klinischer Bedeutung ist, dass bei Patienten mit NOA die Leydigzellfunktion für den mTESEErfolg eine geringfügigere Rolle zu spielen scheint als bei den Patienten mit KS. Dass die Höhe des Testosteronspiegels zum Zeitpunkt der Operation beim KS für das mTESE-Ergebnis relevant ist, deckt sich mit bisher bekannten Daten [3].

Zu loben ist, dass im Rahmen der Studie grundsätzlich die Ergebnisse bei der Verwendung kryokonservierter Spermien verglichen wurden, d.h. keine Mischpopulation von frischen und kryokonservierten Spermien verwendet wurde.

Eine Schwäche der Studie liegt in der Tatsache, dass die Prozedur der mikroskopischen Spermienasservierung durch verschiedene Chirurgen durchgeführt wurde, die unterschiedlich in ihrem Ausbildungsstand und ihren Fertigkeiten waren.

Aus den publizierten Studiendaten ist nicht ersichtlich, ob in der Gruppe der KSPatienten, die einen subnormalen Testosteronspiegel zum Zeitpunkt der mTESE hatten, eine vorherige Testosteronsubstitution durchgeführt wurde. Insbesondere bleibt unklar, ob eine eventuelle Testosterontherapie zuvor abgesetzt wurde. Da exogen zugeführtes Testosteron die gonadotrope Stimulation der Hoden supprimiert, würde bei einer solchen Behandlung vor der mTESE auch nach dem Absetzen möglicherweise noch keine ausreichende Zeit für eine Regeneration der Achse gegeben sein, was sich negativ auf die Spermienausbeute im Rahmen einer mTESE auswirken würde.

Weiterhin ist offen, ob bei Patienten eine Stimulation der Leydigzellfunktion mit hCG, wie von Ramasamy 2009 [4] vorbeschrieben, oder mit Aromataseinhibitoren erfolgte, die möglicherweise die mTESE-Ergebnisse verbessern könnten.
Es wurden keine Jugendlichen in die Untersuchung aufgenommen. Eine solche Untersuchung wäre sinnvoll gewesen, zumal es sich beim Klinefelter-Syndrom um einen progressiv degenerativen Prozess der Hoden handelt und daher anzunehmen ist, dass eine früh durchgeführte mTESE mit anschließender Kryokonservierung die Erfolgsrate einer mTESE von ca. $30 \%$ positiv beeinflusst hätte [5].

Für den Praktiker ist es interessant zu wissen, dass ein jüngeres Lebensalter die Chancen beim KS steigert, Spermien aus den Hoden mittels mTESE zu extrahieren, wohingegen dies nicht bei Männern mit NOA der Fall ist. Somit ist es vorteilhaft, Patienten mit Kinderwunsch und KS frühzeitig an einen erfahrenen Andrologen zur Beratung zu verweisen, damit die Chancen auf Vaterschaft optimal ausgeschöpft werden. Wichtig ist auch die Erkenntnis, dass die endokrine Funktion beim KS wegweisend für den mTESE-Erfolg zu sein scheint.

Abschließend bleibt offen, welche prädiktiven Parameter ansonsten verwendet werden könnten, um vor Durchführung einer mTESE beim KS prognostische Aussagen zum Erfolg einer Spermienextraktion zu treffen. Dies sollte Gegenstand zukünftiger Forschung sein, ebenso wie die Frage, inwiefern der degenerative Prozess der Hoden beim Patienten mit KS aufhaltbar ist.

Dr. Julia Rohayem, Prof. Dr. Sabine Kliesch, Münster

\section{Literatur}

1 Aksglaede L, Juul A. Testicular function and fertility in men with Klinefelter syndrome: a review. Eur J Endocrinol 2013; 168: R67R76

2 Dabaja AA, Schlegel PN. Microdissection testicular sperm extraction: an update. Asian J Androl 2013; 15: 35-39

3 Sabbaghian M, Modarresi T, Hosseinifar H et al. Comparison of sperm retrieval and intracytoplasmic sperm injection outcome in patients with and without Klinefelter syndrome. Urology 2014; 83: 107-110

4 Ramasamy R, Ricci JA, Palermo GD et al. Successful fertility treatment for Klinefelter's syndrome. J Urol 2009; 182: 1108-1113

5 Mehta A, Paduch DA. Klinefelter syndrome: an argument for early aggressive hormonal and fertility management. Fertil Steril 2012; 98: 274-283 


\section{Bildgebende Kontrollen bei Erwachsenen}

Bei Korrektur einer Ureterabgangsstenose nach AndersonHynes resultieren im Allgemeinen gute Ergebnisse, mit Erfolgsraten von mehr als $90 \%$. In den letzten Jahren kommen dabei zunehmend minimalinvasive Verfahren zum Einsatz, mit anscheinend ähnlichen Erfolgs- und Komplikationsraten wie beim offenen Zugang. Die Kontrolle erfolgt dabei meistens klinisch und radiologisch, aber wie lange nach dem Eingriff die Bildgebung fortgesetzt werden sollte, ist unklar. J Urol 2014; 191: 1357-1362

\section{mit Kommentar}

Nach Nierenbeckenplastik im Erwachsenenalter erfolgt ab Jahr 1 nach dem Eingriff oft keine bildgebende Kontrolle mehr. Das haben Ryan Hsi und seine Mitarbeiter festgestellt, die Befunde von insgesamt 742 Patienten retrospektiv ausgewertet haben.

Die Wissenschaftler aus Seattle zogen dazu eine US-weite Datenbank heran, die ca. 50\% aller aus US-amerikanischen Kliniken entlassenen Patienten umfasst (MarketScan). In die Auswertung eingeschlossen wurden Patienten ab dem 18. Lebensjahr, die zwischen 2007 und 2010 wegen einer Ureterabgangsstenose operativ versorgt - offen oder minimalinvasiv - und mindestens 24 Monate nachbeobachtet worden waren.

Die Mediziner analysierten dann, welche bildgebenden Verfahren in welchen zeitlichen Abständen zum Eingriff durchgeführt worden waren. Dabei wurden funktionelle Untersuchungen (Ausscheidungsurogramm, Nierenfunktionsszintigrafie) und rein bildgebende Verfahren (CT, MRT, Sonografie) unterschieden. Die Zeiträume wurden kategorisiert als bis Monat 6, Monat 6-12, Monat 12-24, Monat 24-36 und ab Monat 36.

Die mittlere Nachbeobachtungszeit der eingeschlossenen 742 Patienten betrug etwas mehr als 3 Jahre, knapp zwei Drittel von ihnen (65\%) waren minimalinvasiv operiert worden. Bei $88 \%$ war postinterventionell mindestens 1 -mal ein bildge- bendes Verfahren eingesetzt worden, bei $34 \%$ nur innerhalb der ersten 12 Monate, bei knapp der Hälfte $(48,5 \%)$ sowohl vor als auch nach Monat 12 und bei 5,5\% erstmals nach dem ersten postoperativen Jahr. 12\% wurden zu keinem Zeitpunkt mittels bildgebender Verfahren nachuntersucht.

Innerhalb der ersten 6 Monate wurde bei 554 Patienten $(75 \%)$ mindestens eine bildgebende Untersuchung durchgeführt, innerhalb der ersten 12 Monate war das bei $82 \%$ der Fall, in der Regel waren dies Funktionsuntersuchungen, meistens ein Nierenszintigramm. Zwischen Monat 12 und Monat 24 wurden häufiger rein bildgebende Untersuchungen durchgeführt, meistens eine Sonografie oder CT.

Die multivariate Analyse ergab eine höhere Wahrscheinlichkeit für mindestens 1-mal jährliche bildgebende Untersuchungen bei Frauen (Odds Ratio [OR] 1,48 vs. Männer), älteren Patienten (OR 1,41 für Alter 50-63 Jahre vs. 17-39 Jahre) und längerem Klinikaufenthalt beim primären Eingriff (OR 1,92 für $\geq 6$ Tage vs. $\leq 2$ Tage).

Bei 62 Patienten wurden insgesamt 65 Revisionseingriffe notwendig, im Mittel 10,9 Monate nach der ersten Intervention. Dabei wurde in 27 Fällen eine Drainage eingelegt (44\%) und in jeweils 19 Fällen (31\%) eine Rezidiv-Nierenbeckenplastik bzw. eine Nephrektomie durchgeführt.

\section{Fazit}

Zeitpunkt und Art der bildgebenden Kontrollen nach Nierenbeckenplastik schwanken bei Erwachsenen deutlich, meine die Autoren - nur bei etwa der Hälfte der Patienten werden ab Monat 12 noch entsprechende Untersuchungen durchgeführt. Das könnte, so Hsi et al. weiter, zu einer Überschätzung der tatsächlichen Erfolgsraten führen, denn Spätkomplikationen auch noch 2 Jahre und mehr nach der Operation sind durchaus beschrieben. Vor allem jüngere Männer, die nur kurz in der Klinik gewesen waren, sind von dieser „Unterdiagnostik“ betroffen. Zukünftige Studien sollten die Gründe dieser Unterschiede klären, weiterhin sollten einheitliche Standards für die postinterventionellen Kontrollen erarbeitet werden - so die Autoren.

Dr. Elke Ruchalla, Trossingen

\section{Kommentar}

\section{Follow-up nach} Pyeloplastik im Erwachsenenalter zu kurz?

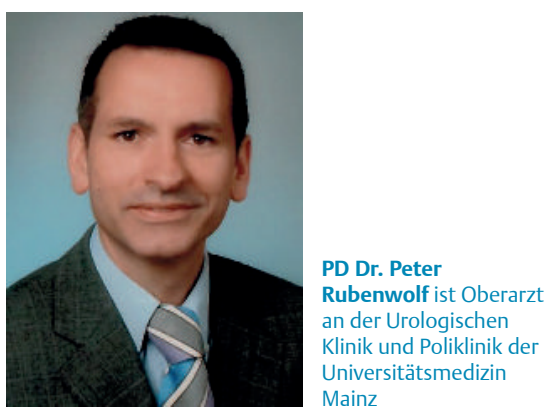

Die kontinuitätsdurchtrennende Pyeloplastik ist seit ihrer Erstbeschreibung durch Anderson und Hynes 1949 ein weltweit durchgeführtes, für alle Patientenalter geeignetes, etabliertes Operationsverfahren, dessen ausgezeichnete Ergebnisse in zahlreichen Publikationen objektiviert werden konnten. Auch den konventionell-laparoskopischen und robotisch assistierten Techniken wird ein vergleichbar gutes Outcome wie der offen-operativen Methode attestiert [1,2].

Es stellt sich insofern zu Recht die Frage, ob es weiterer Arbeiten bedarf, die die 
Langzeitergebnisse der Pyeloplastik de novo auf den Prüfstand stellen, und in diesem Kontext, ob die bislang uneinheitlich gehandhabte Praxis der Nachsorge mittels bildgebender Verfahren einer kritischen Reevaluierung bedarf. Brauchen wir ganz im Trend der zunehmend von Leitlinien geprägten Medizin - nun auch ein Schema für die Nachsorge nach Pyeloplastik?

Hsi und Kollegen kommen in ihrer Arbeit zu dem Ergebnis, dass lediglich 52\% der untersuchten erwachsenen Studienpopulation nach Pyeloplastik $(\mathrm{n}=742)$ ab dem 2. postoperativen Jahr mittels eines bildgebenden Verfahrens nachgesorgt wurden. Sie schlussfolgern daraus, dass die gemeinhin als hoch eingeschätzten (Langzeit-) Erfolgsraten der Pyeloplastik einer kritischen Überprüfung nicht standhalten, und insbesondere, dass asymptomatische Therapieversager nicht rechtzeitig entdeckt würden.

Während sich die Stärken der Arbeit in der stattlichen Patientenzahl und ihrer per se interessanten Fragestellung erschöpfen, sind nachfolgende Aspekte im Rahmen einer kritischen Würdigung der Ergebnisse und Schlussfolgerungen zu bedenken:

1. Eine allgemein anerkannte Definition des Terminus „OP-Erfolg“ nach Pyeloplastik fehlt, sodass die Vergleichbarkeit der publizierten Ergebnisse der Methode ohnehin limitiert ist. Es gilt jedoch zu bedenken, dass die Indikation zur Pyeloplastik beim Erwachsen im Gegensatz zum Kindesalter praktisch ausschließlich aufgrund einer symptomatischen Obstruktion gestellt wird. Die primäre Zielsetzung des Eingriffs liegt daher nicht in einer Verbesserung radiomorphologischer oder nuklearmedizinischer Parameter mit renoprotektiver Intention begriffen, sondern in einer Beseitigung klinischer Symptome. Wenngleich therapiewürdige Rezidivobstruktionen nach Pyeloplastik auch nach dem 1. postoperativen Jahr auftreten können, so sind diese nahezu ausschließlich symptomatischer Natur und somit keine primären Befunde der Bildgebung [3]. De facto wird die Rate asymptomatischer Rezidive in der Literatur lediglich mit etwa $2 \%$ angegeben [4]. Die Rationale einer funktionellen (MAG III / IVP) oder nicht funk- tionellen Bildgebung (Sonografie) aller asymptomatischen adulten Patienten über das 1. postoperative Jahr hinaus ist aus diesem Grund weder medizinisch noch ökonomisch zu vertreten, sondern ist allenfalls akademischen Fragestellungen vorbehalten.

2. Hsi et al. geben eine Rate von $8,4 \%$ sekundärer Prozeduren aufgrund postoperativer Komplikationen an; eine Rezidivpyeloplastik wurde bei 19 von 742 Patienten (2,5\%) durchgeführt und eine ebenso große Patientenzahl einer Nephrektomie unterzogen. Die jeweiligen Indikationen für die durchgeführten Sekundäreingriffe werden allerdings nicht angegeben. Entscheidend im Kontext der Schlussfolgerung ist zudem, dass Zweiteingriffe aufgrund einer Obstruktion durchschnittlich nach 10,9 Monaten durchgeführt wurden, also im ersten Drittel des gesamten Beobachtungszeitraums. Interessant wären hier detaillierte Angaben zu Rezidiv-/Sekundäreingriffen nach Ablauf des ersten, zweiten oder dritten postoperativen Jahres. Insofern ist die Schlussfolgerung der Autoren nicht überzeugend.

3. Die der Studie zugrunde liegende „MarketScan ${ }^{\circledR}$ database“ lässt einen Selektionsbias vermuten, sodass die Ergebnisse und insbesondere die Übertragbarkeit auf europäische Verhältnisse mit der gebotenen Vorsicht interpretiert werden müssen.

4. Unter anderem wurden CT und MRT als bildgebende Verfahren im Rahmen der Nachsorge verwendet; deren Stellenwert nach Pyeloplastik ist bestenfalls zweifelhaft.

\section{Fazit für die Praxis}

Zusammenfassend lässt sich schlussfolgern, dass die Arbeit von Hsi et al. gemeinhin bekanntes Wissen, nämlich hohe Erfolgsraten der Pyeloplastik von über 90\%, replizieren. Weiterhin, dass Sekundäreingriffe aufgrund persistierender oder rezidivierender Obstruktionen in der überwiegenden Zahl der Fälle innerhalb eines Jahres nach dem Eingriff auftreten.

Aus akademischer Sicht ist die Arbeit insofern von Interesse, als die in der Literatur publizierten Langzeitergebnisse nach Pyeloplastik möglicherweise nicht in al- len Fällen ausreichend objektivierbar sind und zudem der Terminus „OP-Erfolg“ nicht einheitlich definiert ist.

Aus klinisch-praktischer Sicht hingegen ist die Studie wenig hilfreich. An der Urologischen Universitätsklinik Mainz überblicken wir die Verläufe von über 900 durchgeführten Pyeloplastiken in den letzten 20 Jahren. Patienten, die postoperativ asymptomatisch sind bzw. bleiben und gleichzeitig in der Sonografie eine Abnahme der Nierenbeckenkelchdilatation im Vergleich zu präoperativ aufweisen, bedürfen unserer Ansicht nach weder einer dynamische Nierenfunktionsszintigrafie (MAG3) noch serieller Nephrosonografien über das erste postoperative Jahr hinaus. Es versteht sich von selbst, dass im Falle unklarer Befunde Verlaufskontrollen in individuell festzulegenden Intervallen und ggf. auch Langzeitkontrollen in jährlichen Abständen indiziert sind.

\section{PD Dr. Peter Rubenwolf, Mainz}

\section{Literatur}

1 Schwentner C, Pelzer A, Neururer R et al. Robotic Anderson-Hynes pyeloplasty: 5-year experience of one centre. BJU Int 2007; 100: 880-885

2 Fedelini P, Verze P, Meccariello C et al. Intraoperative and postoperative complications of laparoscopic pyeloplasty: a single surgical team experience with 236 cases. J Endourol 2013; 27: 1224-1229

3 Madi R, Roberts WW, Wolf JS Jr. Late failures after laparoscopic pyeloplasty. Urology 2008; 71: 677-680

4 Dimarco DS, Gettman MT, McGee SM et al. Long-term success of antegrade endopyelotomy compared with pyeloplasty at a single institution. J Endourol 2006; 20: 707-712

Deutsche Kontinenz Gesellschaft

\section{Neuer Vorstand}

Auf ihrem Jahreskongress wählte die Deutsche Kontinenz Gesellschaft in Frankfurt einen neuen Vorstand. Dabei schien das Motto des Kongresses Pate zu stehen „Tradition und Innovation“.

Prof. Klaus-Peter Jünemann scheidet nach 11-jähriger Arbeit als Erster Vorsitzender aus. Er trat nicht mehr zur Wahl an. Sein Nachfolger wird Prof. Axel Haferkamp, Direktor der Klinik für Urologie und Kinderurologie am Universitätsklinikum Frankfurt. 
Prof. Klaus-Peter Jünemann hat die Deutsche Kontinenz Gesellschaft weiter in die Öffentlichkeit geführt. Unter seiner Ägide entstanden ein professioneller Internetauftritt sowie aufrüttelnde TV-Spots. Auch die jährlich stattfindende World Continence Week wurde in seiner Amtszeit eingeführt, um Betroffene zu ermutigen, sich ärztliche Hilfe zu suchen. Die Qualität der Behandlung stand ebenfalls in seinem Fokus. Unter anderem resultierte daraus die Initiierung einer Fortbildungsreihe für Pflegepersonal. In enger Kooperation mit Prof. Kölbl wurde zudem die Zertifizierung der Kontinenz- und Beckenboden-Zentren durch die Deutsche Kontinenz Gesellschaft vorangebracht.

Sein Nachfolger, Prof. Axel Haferkamp, wird die Verhandlungen mit weiteren medizinischen Fachgesellschaften (Coloproktologie, Gynäkologie, Urologie) zur gemeinsamen einheitlichen Zertifizierung dieser Zentren weiterführen. „Die erfolgreiche Arbeit der Deutschen Kontinenz Gesellschaft hat den Betroffenen eine Stimme gegeben. Eine unserer vordringlichen Aufgaben ist es jetzt, die interdisziplinäre Behandlungsqualität weiter zu steigern. Dazu gehört die Bündelung unserer Bemühungen gemeinsam mit weiteren Fachgesellschaften. So erhalten wir auch in der Politik Gehör.“

Ohne die Politik, die das letzte Wort bei Kostenübernahmen und Anerkennung von Verfahren hat, könne immer nur punktuell gearbeitet werden. Als wissenschaftliche Fachgesellschaft sei es das Ziel, das Angebot einer individuellen Therapie als Standard zu etablieren. „Dazu gehört auch unser Vorstoß zur Einführung einer Kontinenzschwester - denn das ärztliche Personal kann dieses absehbar steigende Arbeitsaufkommen gar nicht alleine bewältigen“, betont Haferkamp.

Gemeinsam mit Prof. Axel Haferkamp steht Prof. Christl Reisenauer, Leitende Oberärztin Urogynäkologie der Frauenklinik der Universitätsklinik Tübingen, an der Spitze der Deutschen Kontinenz Gesellschaft. Sie löst Prof. Heinz Kölbl als Zweiten Vorsitzenden ab. Mit Prof. Christl Reisenauer sind nun auch die Frauen mit einer Expertenstimme im Vorstand vertreten - denn jede vierte Frau ist von Inkontinenz betroffen.

Nach einer Pressemitteilung (Deutsche Kontinenzgesellschaft) 
Prostatakarzinom

\section{Ungerechtfertigte Werbung für IRE}

Unter dem Titel „Die neue Waffe gegen

Prostatakrebs“ berichtet das Wochenmagazin „Focus“ in der Print-Ausgabe 5/ 2015 über eine neue Methode zur Behandlung des Prostatakrebses, bei der angeblich „mit ultrakurzen Stromstößen Tumorzellen zerstört werden können“. Die sogenannte „Irreversible Elektroporation“ (IRE) sei eine „schonende“ Methode, die beim Prostatakrebs Potenz und Schließmuskelfunktion nicht beeinträchtige und bei der „die Harnröhre ebenso erhalten bleibt wie die Erektionsfähigkeit“, heißt es weiter in dem Artikel. Die Deutsche Gesellschaft für Urologie e. V. (DGU) stellt klar, dass diese Hoffnung machende Werbung für eine „neue Technik im Kampf gegen Prostatakrebs“ ungerechtfertigt und gefährlich ist, da der Wert dieser Behandlung noch völlig ungesichert ist.

„Zur Therapie von Prostatakrebs mittels dieser Methode finden sich in wissenschaftlichen Literaturdatenbanken lediglich 18 Publikationen. Diese beschreiben die technischen Grundlagen der Methodik oder Protokolle neuer Studien, die sich noch in Planung befinden“, sagt Prof. Oliver Hakenberg, Generalsekretär der Deut- schen Gesellschaft für Urologie und Direktor der Klinik für Urologie der Universität Rostock. „Lediglich zwei Arbeitsgruppen beschreiben kurzfristige Behandlungsergebnisse, aber auch nur bezüglich der Durchführbarkeit und der Häufigkeit kurzfristiger Behandlungsnebenwirkungen“, so der Generalsekretär. Mittel- oder langfristige Ergebnisse zur Wirksamkeit und zu Nebenwirkungen liegen nicht vor.

„Niemand kann daher zum gegenwärtigen Zeitpunkt irgendeine Aussage dazu machen, ob diese Methode überhaupt in der Lage ist, einen Prostatakrebs therapeutisch zu beeinflussen, noch viel weniger ist klar, ob sie anderen Behandlungsmethoden überlegen ist. Lediglich die denkbare, aber unbewiesene Vorstellung, dass diese alternative Therapie Prostatakrebszellen zerstören könne, darf keinesfalls Grundlage dafür sein, Patienten Hoffnungen zu machen“, ergänzt Prof. Kurt Miller, 1. DGU-Vizepräsident und Direktor der Urologischen Klinik der Charité, Berlin. Zudem ist diese alternative Behandlungsmaßnahme mit hohen Kosten verbunden, die aufgrund des gänzlich fehlenden Nutzennachweises nicht von den Krankenkassen getragen werden.

„Bei Neuzulassung eines Medikaments gelten heute strengste Anforderungen an den Nachweis eines Nutzens durch Studi- en. Es muss sogar der „Zusatznutzen“ im Vergleich zu bestehenden Therapien belegt werden. Bei medizinisch-technischen Behandlungsverfahren ist das anders. Hier werden solche Ansprüche nicht eingefordert. Allein die technische Durchführbarkeit wird als ausreichend angesehen, um die Anwendung am Menschen zu erlauben“, sagt Prof. Jürgen Gschwend vom DGU-Ressort Leitlinien und Qualitätssicherung und Direktor der Klinik für Urologie der Technischen Universität München.

Für die Experten der DGU steht fest: Die Problematik des Prostatakarzinoms mit möglichen Auswirkungen mancher Behandlungen auf die individuelle Lebensqualität darf nicht dazu Anlass geben, Hoffnungen zu wecken, die durch keinerlei Daten und Fakten gestützt werden. Eine seriöse Berichterstattung in den Medien sollte sich immer über ihre Wirkung im Klaren sein: Wie im hier kritisierten Fall könnten Patienten möglicherweise zu Therapien verleitet werden, die ihnen nichts nützen und bei denen ernsthafte Nebenwirkungen auftreten können. Noch fataler aber, wenn durch die mit falschen Hoffnungen verknüpfte Anwendung der Methode tatsächlich wirksame Behandlungen hinausgezögert werden und dann für manchen Patienten zu spät kommen.

Nach einer Pressemitteilung (DGU) 


\section{Höchstes Risiko nach radikaler Zystektomie}

Obwohl tiefe Venenthrombosen und Lungenembolien

vergleichsweise selten nach urologischen Eingriffen auftreten, haben doch bestimmte Patientengruppen ein erhöhtes Risiko für venöse Thromboembolien. Eine retrospektive US-amerikanische Studie hat nun gezeigt, dass die postoperative Wahrscheinlichkeit venöser Thromboembolien stark abhängig von der Art des Eingriffs ist. J Urol 2014; 192: 793-797

mit Kommentar

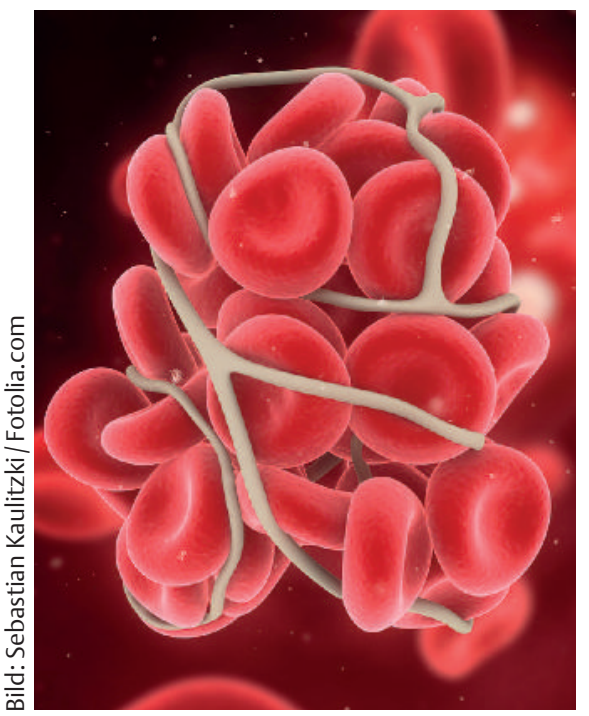

Mark D. Tyson, Urologische Abteilung der Mayo Clinic in Arizona, USA, und Kollegen werteten die NSQIP-Daten von Patienten aus, die sich zwischen Januar 2005 und Dezember 2011 einem urologischen Eingriff unterzogen. Das NSQIP (National Surgical Quality Improvement Program) sammelt klinische Daten von Patienten in über 200 Krankenhäusern und erfasst mehr als 130 Parameter, wie z.B. präoperative demografische Daten, Komorbiditäten, intra- und perioperative Komplikationen und die Mortalität bis 30 Tage nach dem Eingriff.

\section{Risiko gleich null bei laparoskopischer Kolpopexie}

Insgesamt unterzogen sich 82808 Patienten in genanntem Zeitraum einer urologischen Operation. Eine postoperative tiefe Venenthrombose innerhalb von 30 Tagen entwickelten 0,8\% der Patienten ( $\mathrm{n}=633$ ), eine Lungenembolie $0,4 \%(n=349)$. Das höchste Risiko für eine tiefe Venenthrombose hatten Patienten nach radikaler Zystektomie (4,0\%), gefolgt von partieller Zystektomie $(2,4 \%)$ und offener radikaler Nephrektomie (1,7\%). Das geringste Risiko hatten Patienten nach einer laparoskopischen Kolpopexie (0,0\%) sowie nach Schlingenplastiken bei weiblicher Harninkontinenz (0,1\%). Die meisten Lungenembolien $(2,9 \%)$ traten nach einer Zystektomie auf.

Waren die Patienten älter, männlich, hatten einen höheren BMI, einen geringeren funktionellen Status und disseminierte Metastasen, entwickelten sie mit einer höheren Wahrscheinlichkeit eine tiefe Venenthrombose. Diabetes, Raucherstatus oder ethnische Herkunft hatten laut der Studie keinen Einfluss auf das Risiko. Im Median dauerte es 11 Tage bis eine tiefe Venenthrombose postoperativ auftrat, bei einer Lungenembolie 9 Tage.

Die stärksten Prädiktoren für eine venöse Thromboembolie waren

- der funktionelle Status des Patienten (OR 5,59, 95\% KI 3,54-8,82, p<0,001),

- eine frühere kongestive Herzinsuffizienz (OR 4,57, $95 \%$ KI 2,80 - 7,47, $\mathrm{p}<0,001$ ) und

- fortgeschrittene Tumorerkrankung mit disseminierten Metastasen (OR 3,12, $95 \%$ KI 2,48-3,93, p<0,001).

Zudem war ein Alter über 60 Jahre, eine Anästhesiedauer von mehr als $120 \mathrm{~min}$ und eine chronische Steroideinnahme mit der Entwicklung einer venösen Thromboembolie assoziiert.

\section{Fazit}

Die aktuelle Studie ist laut Aussage der Autoren die erste, die einen umfassenden Vergleich von Risiken in Bezug auf venöse Thromboembolien nach urologischen Eingriffen vornimmt. Mit dem Outcome der Studie könne ein Beitrag zur Prävention von Lungenembolien und tiefen Venenthrombosen geleistet werden, so die Autoren. Sie weisen aber auch auf die Limitationen ihrer Studie hin: Sie erfassten die Daten retrospektiv und es lagen keine Angaben für eine etwaige Thromboembolieprophylaxe vor. Wünschenswert sei außerdem ein postoperatives Follow-up von 90 Tagen gewesen.

Dr. Antonie Post, Burgstetten

\section{Kommentar}

\section{Differenzierte}

Erkenntnisse für die Urologie
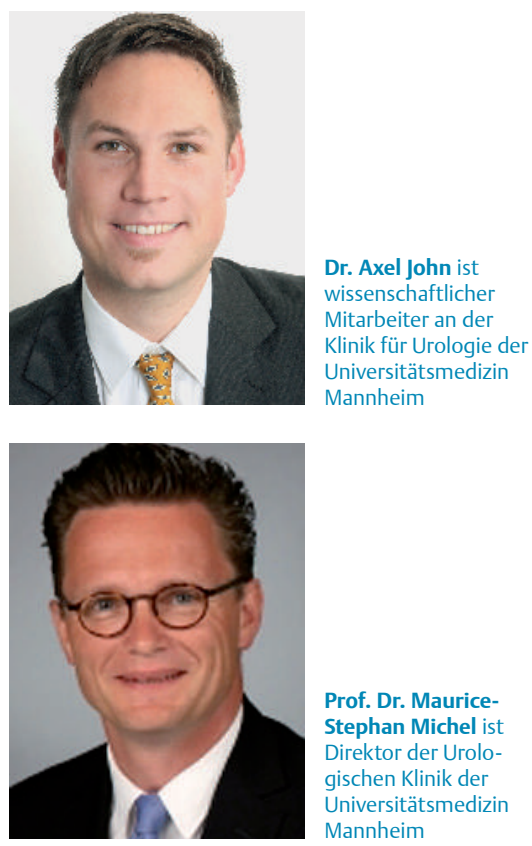

Prof. Dr. MauriceStephan Michel ist Direktor der Urologischen Klinik de Universitätsmedizin Mannheim

Tyson und Kollegen zeigen in ihrer Studie anhand eines großen Kollektivs aktuelle Daten über die 30-Tages-Inzidenz von VTE nach urologischen Eingriffen. Hierbei betonen Sie die im Vergleich zu vorherigen Publikationen präzise Differenzierung der unterschiedlichen Operationen. Zum anderen erlaubt die Studienpopulation von knapp 83000 Patienten mit jeweils um- 
fangreicher Charakterisierung ( $>130$ bekannte Variablen/Patient) eine aussagefähige multivariate Analyse im Hinblick auf Risikofaktoren für VTE [1].

Die aktuellen S3-Leitlinien zur VTE-Prophylaxe in Deutschland empfehlen eine Einstufung der Patienten in Gruppen mit geringem, mittleren oder hohem VTE-Risiko. Dafür müssen expositionelle (aus dem Eingriff resultierende) und dispositionelle (bei dem Patienten individuell vorliegende) Risikofaktoren bedacht werden [2]. Die vorliegende Arbeit liefert hierfür eine breite Datenbasis mit detaillierten Angaben für verschiedene urologische Eingriffe. Zusätzlich werden bereits bekannte dispositionelle Risikofaktoren (Vorherige VTE, Alter $>60$ Jahre, Thrombophilie, aktive Malignom-Erkrankung, Sexualhormonmanipulation, Chronische Herzinsuffizienz, BMI > 30, Infekt mit Immobilisation) um die statistisch signifikanten Prädiktoren schlechter Allgemeinzustand, Steroidtherapie und Dauer der Anästhesie > 2 Stunden ergänzt.

Ein interessanter Punkt der vorliegenden Arbeit sind die unterschiedlichen VTE-Raten bei offenen vs. laparoskopischen Eingriffen. Auch wenn nicht explizit statistisch verglichen, so erscheinen unter den 10 Eingriffen mit der höchsten VTE-Rate (Zystektomie mit 3,96\% bis zu offener partieller Nephrektomie mit 0,97\%) bis auf die „offene oder laparoskopische Steinsanierung“ keine laparoskopischen Interventionen. Somit scheint hier ein Anhalt für eine möglicherweise geringere VTERate in der laparoskopischen urologischen Chirurgie zu bestehen. In den Leitlinien wird bisher keine Differenzierung zwischen offener und laparoskopischer Chirurgie betrieben. Aufgrund der hohen Kosten sowie der potenziellen Risiken der VTE-Prophylaxe (z.B. heparininduzierte Thrombozytopenie) käme einer genaueren Unterscheidung eine hohe Relevanz zu. Aufgrund der einer retrospektiven Studie inhärenten Einschränkungen in Bezug auf Selektionsbias und andere Confounder sollte hier jedoch nur von einer Hypothese gesprochen werden und nicht von einem wissenschaftlichen Nachweis. Weitere Studien diesbezüglich wären wünschenswert.

Kritisch zu werten ist der mit 30 Tagen relativ kurze Beobachtungszeitraum der Studie, wodurch möglicherweise einige späteVTEübersehen wurden. Nichtsdestotrotz wissen wir aus ähnlichen Arbeiten, dass z.B. für die radikale Prostatektomie die Mehrzahl der VTE innerhalb der ersten 4 Wochen postoperativ auftritt [3]. Ein Schwachpunkt dieser Arbeit sind die fehlenden Angaben zur perioperativen VTEProphylaxe. Prinzipiell stehen hier dem behandelnden Arzt medikamentöse (z.B. Heparin) und komplementäre (z.B. Frühmobilisation, adäquate Hydrierung, Kompressionsstrümpfe) Maßnahmen zur Verfügung. Die Adhärenz zu den gängigen Leitlinienempfehlungen hatte vermutlich einen starken Einfluss auf die Studienergebnisse und wurde leider nicht erfasst. So ist es erstaunlich, dass die TVT-Rate (TVT: Tiefe-Venen-Thrombose) nach radikaler Zystektomie mit einem Wert von 3,96\% in der vorliegenden Studie ähnlich den Ergebnissen aus den 90er Jahren sind (3,7\%), einer Zeit als die VTE-Prophylaxe noch nicht flächendeckend durchgeführt wurde [4]. Bezüglich der Interpretation der Daten für den deutschsprachigen Raum ist anzumerken, dass in den USA die VTE-Prophylaxe in der Regel erst postoperativ beginnt, während in Deutschland die Heparin-Erstgabe meist am Vorabend der OP erfolgt. Des Weiteren werden in den USA einige Eingriffe, die hierzulande regelhaft unter stationären Bedingungen erfolgen, ambulant durchgeführt (z.B. die URS).

Für die Praxis liefert die vorliegende Arbeit eine gute Datenbasis für die Einschätzung des VTE-Risikos gängiger urologischer Eingriffe und identifiziert einen schlechten Allgemeinzustand, Steroidtherapie und Dauer der Anästhesie > 2 Stunden als zusätzliche unabhängige Risikofaktoren für VTE.

\section{Dr. Axel John, Prof. Dr. Maurice-Stephan Michel, Mannheim}

\section{Literatur}

1 Tyson MD, Castle EP, Humphreys MR et al. Venous Thromboembolism after Urologic Surgery. J Urol 2014; 192: 793-797

2 Arbeitsgemeinschaft der Wissenschaftlichen Medizinischen Fachgesellschaften. S3Leitlinie: Prophylaxe der venösen Thromboembolie (VTE). 18. 03. 2009. Im Internet: http://www.awmf.org/uploads/tx_ szleitlinien/003-0011_S3_ThromboembolieProphylaxe_2010_01.pdf

3 Van Hemelrijck M, Garmo H, Holmberg L et al. Thromboembolic events following surgery for prostate cancer. Eur Urol 2013; 63: 354-363

4 White RH, Zhou H, Romano PS. Incidence of symptomatic venous thromboembolism after different elective or urgent surgical procedures. Thromb Haemost 2003; 90: 446455
Erektile Dysfunktion

\section{Gute Blutzucker- einstellung schützt}

Diabetes mellitus ist die häufigste Ursache für Potenzstörungen - jeder zweite männliche Diabetespatient leidet unter Erektionsproblemen. Die beste Therapie, die Potenz zu erhalten und eine beginnende Erektionsstörung zu stoppen, ist eine gute Blutzuckereinstellung. Darauf weist die Deutsche Diabetes Gesellschaft (DDG) hin. Wer erste Anzeichen bemerkt, sollte daher seinen Lebensstil überprüfen und erwägen, eine psychologische Beratung in Anspruch zu nehmen.

Ein überhöhter Blutzuckerspiegel schädigt mit der Zeit die Blutgefäße. Die Folgen davon sind Durchblutungsstörungen. Sie machen sich oft zuerst an den Füßen bemerkbar, die sich taub anfühlen und unempfindlich gegenüber Berührungen werden. „Danach stellen sich bei Männern häufig auch Erektionsprobleme ein“, erläutert PD Dr. Erhard Siegel, Präsident der DDG. Bleibt eine ausreichende Erektion in rund $70 \%$ der Versuche aus und halten die Probleme mindestens 6 Monate an, sprechen Ärzte von einer „erektilen Dysfunktion“.

Doch so lange sollten Diabetespatienten keinesfalls warten, bis sie ihren Arzt aufsuchen. „Denn die beste Therapie, die Potenz zu erhalten oder eine beginnende Erektionsstörung nicht schlimmer werden zu lassen, ist eine gute Blutzuckereinstellung“", sagt Siegel. Experten gehen heute davon aus, dass eine schlechte Einstellung auch vorübergehend Erektionsprobleme hervorrufen kann - Patienten können damit selbst aktiv zur Therapie beitragen.

Wie die Blutzuckereinstellung verbessert werden kann, besprechen die Patienten mit dem Diabetologen. Die DDG rät, zunächst den Lebensstil zu überprüfen: Rauchen ist sehr abträglich, übermäßiger Alkoholkonsum ebenfalls. Positiv auf den Blutzuckerspiegel wirken sich hingegen Bewegung, gesunde Ernährung und Gewichtsreduktion aus. „Sehr effektiv kann auch eine Beratung sein, wie der Patient die Blutzuckerkontrolle besser in den Griff kriegt“, fügt Siegel hinzu. Darüber hinaus kann ein Therapiewechsel helfen, die Werte zu normalisieren.

Nach einer Pressemitteilung (DDG) 


\section{Erhöht eine zytoreduktive Nephrektomie das Gesamtüberleben?}

\author{
Klinische Studien haben gezeigt, dass die zytoreduktive Neph- \\ rektomie beim metastasierenden Nierenzellkarzinom als Er- \\ gänzung einer zielgerichteten Therapie die Lebensdauer von \\ Patienten verlängern kann. Eine internationale retrospektive \\ Studie ging nun der Frage nach, ob die zytoreduktive Nephrek- \\ tomie immer Teil des empfohlenen Behandlungsprotokolls sein \\ sollte. Laut Heng et al. ist das Krankheitsstadium von entschei- \\ dender Bedeutung. \\ Eur Urol 2014; 66: 704-710
}

\section{mit Kommentar}

Daniel Y.C. Heng vom Tom Baker Cancer Center Alberta, Kanada, und Kollegen sammelten retrospektiv Patientendaten aus 20 internationalen Tumorzentren in Kanada, USA, Belgien, Südkorea, Japan, Dänemark, Griechenland und Singapur unter Zuhilfenahme des IMDC (International Metastatic Renal Cell Carcinoma Database Consortium). Die IMDC-Prognosefaktoren waren:

- Hämoglobin unterhalb des unteren Grenzwerts des Normalbereichs (Anämie),

- korrigiertes Serumkalzium, neutrophile Granulozyten und Thrombozyten über der Obergrenze des Normalbereichs,

- Karnofsky-Index $<80 \%$ und

- Zeit von Diagnose zur Behandlung < 1 Jahr.

Einschlusskriterien waren die Diagnose eines Nierenzellkarzinoms jeglicher Art mit synchroner Metastasierung plus Therapie mit einem Tyrosinkinaseinhibitor.

Von den 3245 Patienten mit metastasiertem Nierenzellkarzinom der IMDC wurden 79\% nephrektomiert $(n=2569)$. Patienten, die sich einer Nephrektomie vor der Erstdiagnose einer Metastasierung unterzogen, d.h. solche mit einer metachronen Metastasierung, schlossen die Autoren aus ( $n=1587$ ). In die Analyse gingen letztendlich 1658 Patienten ein. Bei $59 \%(n=982)$ ist eine Niere operativ entfernt worden, die restlichen Patienten

( $n=676$ ) hatten keine zytoreduktive Nephrektomie. Das Follow-up betrug im Median 39,1 Monate. Zum Zeitpunkt der Analyse waren 68,6\% der Patienten verstorben $(n=1137)$ und $85,4 \%$ der Patienten hatten einen progressiven Krankheitsverlauf erfahren $(n=1416)$.

\section{Nephrektomie-Patienten hatten insgesamt eine bessere Prognose $\nabla$}

Alle Patienten der Studie hatten eine zielgerichtete Therapie erhalten, die meisten eine First-line-Therapie mit Sunitinib (72\%). Nephrektomie-Patienten hatten eine signifikant bessere Prognose nach IMDC-Kriterien im Vergleich zu Patienten ohne Operation:

> günstig Prognose: 9 vs. $1 \%$

> mittelmäßig: 63 vs. $45 \%$

> schlecht: 28 vs. $54 \%$

In der Nephrektomie-Gruppe hatten weniger Patienten eine unklare Zellpathologie $(p=0,042)$, Knochenmetastasen $(p=0,001)$ und Lebermetastasen $(p=0,001)$, jedoch mehr sarkomatoide Varianten $(p<0,001)$.

Das mediane Gesamtüberleben in der Nephrektomie-Gruppe lag bei 20,6 Monaten, bei Patienten ohne Nephrektomie bei 9,6 Monaten $(\mathrm{p}<0,001)$. Auch nach Anpassung der IMDC-Risikofaktoren hatten die Patienten mit einer zytoreduktiven Nephrektomie einen Vorteil. Die Nephrektomie war zudem assoziiert mit einer höheren pro- gressionsfreien Überlebensrate von 7,6 vs. 4,5 Monate ( $p<0,001)$, die auch nach Anpassung der Prognosefaktoren bestehen blieb. Je höher die Überlebenswahrscheinlichkeit eines Patienten geschätzt wurde, desto vorteilhafter wirkte sich eine $\mathrm{Ne}$ phrektomie auf das Gesamtüberleben aus.

\section{Fazit}

Eine zytoreduktive Nephrektomie könne das Gesamtüberleben bei Patienten mit einem synchron metastasierten Nierenzellkarzinom und zielgerichteter Therapie verlängern, so die Autoren. Lag die geschätzte Überlebensdauer der Patienten jedoch bei weniger als 12 Monaten, so trug eine Nephrektomie nur wenig zu einer Verlängerung des Gesamtüberlebens bei, ebenso bei Patienten, die mindestens 4 der IMDC-Risikofaktoren erfüllten.

Dr. Antonie Post, Burgstetten

\section{Kommentar}

\section{Indikation zur} zytoreduktiven

\section{Tumornephrektomie}

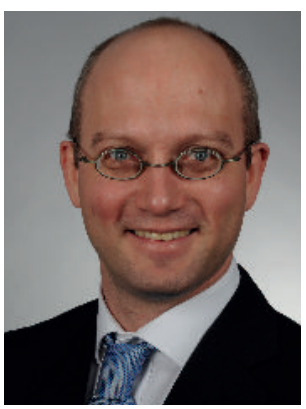

PD Dr. Frederik C.

Roos ist Oberarzt der Urologischen Klinik und Poliklinik der Universitätsmedizin Mainz

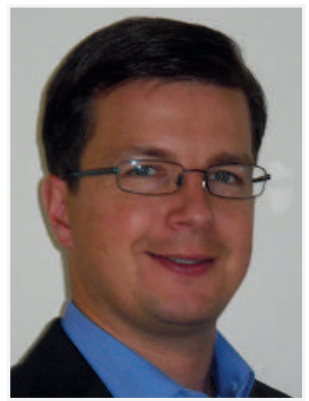

PD Dr. Christian Thomas ist Oberarzt der Urologischen Klinik und Poliklinik der Universitätsmedizin Mainz

Das Nierenzellkarzinom macht 3\% aller soliden Tumoren aus. Synchrone Lymphknoten und/oder Fernmetastasen treten bei ca. 20\% der Patienten auf [1]. Während die Resektion synchron solitärer gut operativ zugänglicher Metastasen einen fes- 
ten Stellenwert in der Therapie des metastasierten Nierenzellkarzinoms einnimmt, wird die Tumornephrektomie im Rahmen einer Zytoreduktion bei synchron multiplen Metastasen in der Ära der Multi-Tyrosinkinaseinhibitoren kontrovers diskutiert. Nach Tumornephrektomie wurden spontane Remissionsraten von pulmonalen Metastasen in bis zu 4\% der Fälle beschrieben [2]. Neben der spontanen Remission werden die Reduktion der Tumormasse und die Vermeidung von lokalen Komplikationen mit Steigerung der Lebensqualität als Begründung für eine zytoreduktive Tumornephrektomie angeführt. In einer Studie von Pierorazio et al. führte eine $90 \%$ ige Reduktion der Tumormasse zu einer Überlebensverlängerung von 11,6 Monaten im Vergleich zu 2,8 Monaten ohne Reduktion der Tumormasse [3].

In der Zytokin-Ära war die zytoreduktive Tumornephrektomie bei gut selektierten Patienten mit einem Performancestatus von 0-2 ein fester Bestandteil des multimodalen Therapiekonzepts $[2,4]$. In den aktuellen Therapiestrategien ist die Immuntherapie durch die besseren Ergebnisse der Tyrosinkinaseinhibitoren in den Hintergrund getreten [5] und der Stellenwert der zytoreduktiven Tumornephrektomie muss neu untersucht und bewertet werden.

Diesen Stellenwert berücksichtigend bietet die Arbeit von Heng et al. eine sehr gute Orientierung zur Konsultation der betroffenen Patienten. Aktuell ist es die größte Studie, die an Patienten $(\mathrm{n}=1658) \mathrm{mit}$ synchron metastasiertem Nierenzellkarzinom durchgeführt wurde. Die Patienten, die sich einer zytoreduktiven Tumornephrektomie $(n=982)$ unterzogen, wurden Patienten $(n=676)$ gegenübergestellt, die nicht operiert wurden. Heng et al. konnten zeigen, dass in der Subgruppenanalyse Patienten mit multiplen Metastasierungsorten, Patienten mit Gehirn-, Knochenund Lebermetastasen von einer zytoreduktiven Therapie profitieren. Ferner war das mediane Gesamtüberleben bei den Patienten mit einem Karnofksy-Performance-Status (KPS) $<80$, bei Patienten $>75$ Jahre und bei Patienten mit einem nicht klarzelligen Karzinom nach zytoreduktiver Tumornephrektomie signifikant besser als bei Patienten, die unter Berücksichtigung dieser Parameter nicht operiert wurden.

Die Arbeit konnte deutlich herausstellen, dass Patienten mit einem ästimierten Ge- samtüberleben von < 12 Monaten weniger von einer zytoreduktiven Therapie profitieren. In Anlehnung an die prognostischen Faktoren des International Metastatic Renal Cell Carcinoma Database Consortium (IMDC) konnte anhand der Cox-Regression gezeigt werden, dass die zytoreduktive Tumornephrektomie das Gesamtüberleben der Patienten mit > 3 Risikofaktoren nicht verbessern konnte.

In einer japanischen Studie wurden retrospektiv 171 Patienten untersucht, von denen 96 Patienten eine zytoreduktive Therapie gefolgt von einer Therapie mit MultiTyrosinkinaseinhibitoren und 75 Patienten nur Multi-Tyrosinkinaseinhibitoren erhielten. Nephrektomierte Patienten mit $<2$ Risikofaktoren nach IMDC hatten einen ca. 10-monatigen Gesamtüberlebensvorteil gegenüber denjenigen, die nur medikamentös behandelt wurden [6]. Zwei kürzlich erschiene Reviews untermauern, dass Patienten mit einem guten Performancestatus von der Operation profitieren werden $[7,8]$.

Die Arbeit um Heng et al. ist retrospektiv und sicherlich mit Selektionsbias behaftet. Vor allem hinsichtlich der Einschätzung des KPS, welcher sehr subjektiv erfolgt. $42 \%$ der Patienten, die nicht operiert wurden, wiesen einen KPS von $<80$ auf, während es in der operierten Gruppe 19\% waren. Die Komorbiditäten, die Einfluss auf die Operabilität und das Gesamtüberleben haben, wurden nicht erfasst. Auch die perioperative Morbidität, findet in der Arbeit keine Berücksichtigung. Kritiker der zytoreduktiven Therapie argumentieren, dass einige Patienten nach der Operation einer systemischen Therapie nicht mehr zugeführt werden können. In den großen prospektiven Studien, die die Interferontherapie nach Tumornephrektomie untersuchten, konnten nur 3 / 162 Patienten keine Anschlusstherapie erhalten und die perioperative Mortalität lag bei 0,08 (SWOG) und 2,4\% (EORTC) $[2,4]$.

In den aktuellen EAU-Leitlinien wird die zytoreduktive Tumornephrektomie als Standard empfohlen, da bei den meisten Zulassungsstudien der Multi-Tyrosinkinaseinhibitoren in $90 \%$ der Fälle eine Tumornephrektomie erfolgte [1]. Um den Stellenwert der Tumornephrektomie genau zu bewerten, laufen aktuell 2 prospektive, randomisierte Studien. In der klinischen Studie, die die Bedeutung der Nephrektomie beurteilen soll (CARMENA; NCT00930033), erhalten Patienten mit ei- nem guten Performance-Status (Eastern Cooperative Oncology Gruppe 0 oder 1) randomisiert eine Nephrektomie gefolgt von der zielgerichteten Therapie mit Sunitinib oder Sunitinib allein. Die sofortige Tumornephrektomie oder die Tumornephrektomie nach Sunitinib wird in einer anderen prospektiven, randomisierten Studie (SURTIME; NCT01099423) untersucht.

Bis uns die Ergebnisse dieser beiden Studien zur Verfügung stehen, bietet die Arbeit von Heng et al. die Möglichkeit, Patienten risikostratifiziert bezüglich einer zytoreduktiven Tumornephrektomie zu beraten. Aus unserer Sicht sollten für die Indikationsstellung zur Operation der Performancestatus, der Patientenwunsch und die Komorbiditäten des Patienten zusätzlich mit einfließen.

\section{PD Dr. Frederik C. Roos, PD Dr. Christian}

Thomas, Mainz

Literatur

1 Ljungberg B, Cowan NC, Hanbury DC et al. EAU guidelines on renal cell carcinoma: the 2010 update. Eur Urol 2010; 58: 398-406

2 Flanigan RC, Salmon SE, Blumenstein BA et al. Nephrectomy followed by interferon alfa$2 \mathrm{~b}$ compared with interferon alfa-2b alone for metastatic renal-cell cancer. N Engl J Med 2001; 345: 1655-1659

3 Pierorazio PM, McKiernan JM, McCann TR et al. Outcome after cytoreductive nephrectomy for metastatic renal cell carcinoma is predicted by fractional percentage of tumour volume removed. BJU Int 2007; 100: 755-759

4 Mickisch GH, Garin A, van Poppel H et al. Radical nephrectomy plus interferon-alfabased immunotherapy compared with interferon alfa alone in metastatic renal-cell carcinoma: a randomised trial. Lancet 2001; 358: 966-970

5 Motzer RJ, Hutson TE, Tomczak P et al. Sunitinib versus interferon alfa in metastatic renal-cell carcinoma. N Engl J Med 2007; 356: 115-124

6 You D, Jeong IG, Song C et al. Analysis of preoperative variables for identifying patients who might benefit from upfront cytoreductive nephrectomy for metastatic renal cell carcinoma in the targeted therapy era. Jpn J Clin Oncol 2015; 45: 96-102

7 Krabbe LM, Haddad AQ, Westerman ME et al. Surgical management of metastatic renal cell carcinoma in the era of targeted therapies. World J Urol 2014; 32: 615-622

8 Bex A, Powles T. Selecting patients for cytoreductive nephrectomy in advanced renal cell carcinoma: who and when. Expert Rev Anticancer Ther 2012; 12: 787-797 


\section{Studie zur \\ Prävention von Wundheilungs- störungen}

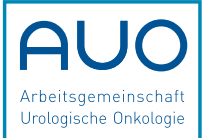

Nach inguinaler Lymphadenektomie kommt es trotz subtiler Präparationstechnik bei bis zu

$50 \%$ der Patienten zu postoperativen Wundheilungsstörungen mit sekundärer Wundheilung, Lymphozelenbildung, persistierender Lymphorrhoe oder Lymphödembildung, die bei bis zu $30 \%$ aufgrund von Schmerzen, Infektion oder Kompression von Gefäßen weiterer Therapie bedürfen [1,2]. Neben einer Minderung der Lebensqualität betroffener Patienten kann dadurch eine Verzögerung der weiteren onkologischen Therapie sowie eine Verschlechterung der Prognose entstehen. Ein unmittelbar postoperativ aufgebrachter epidermaler Vakuumverband kann möglicherweise Lymphgefäße komprimieren, ein Auftreten von Lymphozelen verhindern und auf diese Weise Wundkomplikationen zumindest zum Teil vermeiden helfen. Nach einigen kleineren positiven Studien bei Risikowunden $[3,4]$ inklusive eigenen vielversprechenden retrospektiven Erfahrungen zur Vorbeugung von Wundkomplikationen nach inguinaler Lymphadenektomie [5] soll nun im Rahmen der prospektiven PräVAC-Studie der Nutzen dieser Wundversorgung untersucht werden.

Im Rahmen der PräVAC-Studie (PräVAC: Prävention von Wundheilungsstörungen nach inguinaler Lymphadenektomie bei Patienten mit Peniskarzinom durch epidermale Vakuumtherapie) wird bei Patienten mit Peniskarzinom unmittelbar postoperativ nach beidseitiger inguinaler Lymphadenektomie, Einlage einer subkutanen Redondrainage und Wundverschluss mittels Klammernaht oder Einzelknopfnaht randomisiert auf einer Seite ein konventioneller Druckverband für 24 Std. (Standardversorgung), auf der anderen Seite ein epidermaler Vakuumverband für 7-8 Tage (Intervention) aufgebracht. Als primärer Endpunkt wird geprüft, ob bei mindestens $70 \%$ der Patienten die kumulative Drainagenfördermenge ( $\mathrm{ml}$ ) bis zur Drainagenentfernung (längstens bis zum 14. postoperativen Tag) auf der Seite des Vakuumverbands gerin-

\begin{tabular}{|l|l|}
\hline $\begin{array}{l}\text { Tab.1 Kontaktdaten der Studienzentren } \\
\text { Zentrum }\end{array}$ & Kontakt \\
München & Dr. T. Maurer, Prof. Dr. M. Retz, Prof. Dr. J. E. Gschwend, \\
& Urologische Klinik und Poliklinik der Technischen Universität München, \\
& Ismaninger Str. 22, 81675 München; \\
& 089/4140-25 22, t.maurer@Irz.tum.de \\
\hline Regensburg & PD Dr. H.-M. Fritsche, Dr. S. Schneider, \\
& Klinik für Urologie der Universität Regensburg, \\
& Landshuter Str. 65, 93053 Regensburg; \\
& 0941/782-35 10, hans-martin.fritsche@ukr.de \\
& PD Dr. C. Protzel, \\
& Urologische Klinik und Poliklink der Universität Rostock, \\
& Ernst-Heydemann-Straße 6, 18055 Rostock; \\
& 0381/494-78 01, protzelc@med.uni-rostock.de \\
\hline Rostock &
\end{tabular}

\section{Tab.2 Wichtigste Ein- und Ausschlusskriterien der PräVAC-Studie}

\section{Einschlusskriterien}

Patienten mit Peniskarzinom und Indikation zur inguinalen Lymphadenektomie (ab dem Tumorstadium pT1 G2 oder bei tastbaren Lymphknotenvergrößerungen in der Leiste) ger ist als auf der Gegenseite. Als sekun-
däre Endpunkte werden maximale Drainagenfördermenge pro Tag $(\mathrm{ml})$, Drainagenverweildauer, Auftreten wundassoziierter Komplikationen, Hospitalisationsdauer, Reinterventionsrate sowie die Lebensqualität und Patientenzufriedenheit mit der Wundversorgung untersucht. Die Studie umfasst den Krankenhausaufenthalt des Patienten und endet mit einer Visite 3 Monate postoperativ.

Insgesamt sollen 100 Patienten in dieser von der Urlogischen Klinik und Poliklinik der TU München initiierten Studie eingebracht werden. Der epidermale Vakuumverband (Prevena ${ }^{\mathrm{TM}}$ ) wird im Rahmen der Studie von der Firma KCI GmbH, Wiesbaden, gestellt. Für den primären Endpunkt soll ein exakter Binomialtest auf einem 2 -seitigen Signifikanzniveau von $\alpha=3 \%$ erfolgen, da 2 Zwischenauswertungen (nach 25 und 50 Patienten) auf einem Signifikanzniveau von jeweils $\alpha^{*}=1 \%$ geplant sind.

Die Rekrutierung ist ab sofort an den Studienzentren in München, Rostock und Regensburg ( 0 Tab. 1) möglich, die sich über Patientenzuweisungen zur Studie sehr freuen. Ebenso sind weitere Zentren herzlich willkommen. Die Rekrutierung ist bis Ende 2016 geplant. Die Ein- und Ausschlusskriterien sind in der 0 Tab. 2 ge-

\section{Ausschlusskriterien}

Inguinale Voroperationen (z. B. Z. n. femoraler Bypass-Operation) oder sonst. medizinische Konditionen, die bereits zu einer Behinderung des inguinalen Lymphabflusses geführt haben (Z. n. Leistenherniotomie kein Ausschlusskriterium, sofern OP mehr als 3 Monate zurückliegt und diesbezüglich keine Beschwerden vorliegen wie z. B. gestörter Lymphabfluss)

Patienten mit bekannter Allergie gegen Acrylklebstoffe

listet. Bei Fragen zur Studie wenden Sie sich bitte an den Studienleiter Dr. T. Maurer, 089/4140-2522 (t.maurer@lrz.tum. de) oder an Frau H. Rexer, 039827/79 677 (heidrun.rexer@meckevidence.de).

\section{T. Maurer, C. Protzel, J. E. Gschwend, H. Rexer, Geschäftsstelle der AUO}

\section{Korrespondenz:}

Dr. med. Tobias Maurer, FEBU

Urologische Klinik und Poliklinik

Technische Universität München

Ismaninger Str. 22

81675 München

E-mail: t.maurer@lrz.tum.de

Literatur

1 Stuiver MM, Djajadiningrat RS, Graafland NM et al. Eur Urol 2013; 64: 486-492

2 Pizzocaro G, Algaba F, Horenblas S et al. Eur Urol 2010; 57: 1002-1012

3 Pachowsky M, Gusinde J, Klein A et al. Int Orthop 2011

4 Stannard JP, Atkins BZ, O‘Malley D et al. Ostomy Wound Manage 2009; 55: 58-66

5 Tauber R, Schmid S, Horn T et al. J Plast Reconstr Aesthet Surg 2013; 66: 390-396 